\title{
Effects of Orientation of Carbon Nanotubes on CNT Bundle Based Silk Composite Using Finite Element Method
}

\author{
Md Shihamul Islam*, Jerzy Szpunar \\ Department of Mechanical Engineering, University of Saskatchewan, Saskatoon, Canada \\ Email: ${ }^{*}$ msi875@mail.usask.ca
}

Received November 2, 2012; revised December 5, 2012; accepted December 20, 2012

\begin{abstract}
Carbon Nanotubes (CNTs) have exceptional mechanical properties. They are the stiffest and strongest material, yet discovered, because of their high length to diameter ratio. In this paper a Representative Volume Element (RVE) model of CNT-silk composite is made. Then the orientations of the CNTs in the composite are varied and the effects of this variation are observed. The change is mainly observed between the interfaces of CNT and silk. Also there is a change in Young's Modulus. COMSOL Multiphysics 4.2a is used for building model and performing simulations.
\end{abstract}

Keywords: Carbon Nanotubes (CNTs); Orientation; Interface; Young's Modulus; RVE; FEM; COMSOL Multiphysics 4.2a

\section{Introduction}

Carbon nanotubes (CNTs) can be the ultimate reinforcing materials for the development of nanocomposites because of its extremely high stiffness, strength, and resilience [1]. However, the nanotube dimensions of the order of a few nanometers in diameter and a few hundreds of microns in length have put huge unsolved challenges before researchers. The elastic properties and load carrying capacities of CNTs in nanocomposites have been demonstrated in several research works. Some of these investigations show that the load-carrying capacity of CNTs in a matrix as well as the improvement of the elastic properties of the composites is significant. That is why a lot of different combinations of CNT with different matrix are taken into consideration to observe which combination can provide better mechanical properties. In this paper, the composite that is taken into account is CNT with silk as the matrix.

Different matrix exhibits different types of properties. Most commonly used matrix with CNT is polymer. But now-a-days researchers are looking for naturally available matrix instead of polymer. Silk can play a huge role in this case. Many researchers are looking to make a composite with CNT and silk. They are also interested about the mechanical properties of this kind of composites.

3D silk-carbon nanotube (CNT) based composite scaf-

"Corresponding author. folds is used to acquire better neuron differentiation efficiency from human embryonic stem cells (hESCs) [2]. CNT and silk have been successfully used for neuronal differentiation and tissue engineering applications. The silk-CNT composite scaffolds can serve as efficient support matrices for stem cell derived neuronal transplants that offer a promising opportunity for nerve injury treatments in spinal cord injury (SCI) patients.

Silk consists of two types of proteins-Sericin and Fibroin. Fibroin is the structural center of the silk and Sericin is the sticky material surrounding it. This leads to an interesting aspect about CNT-silk composite which is the interface between them. It is assumed that the CNT and Sericin will most likely to form a bond and create an interface [2]. But more research is necessary to find their actual properties.

It is difficult to get isolated CNT in the production process. CNTs have a propensity to aggregate to bundle or wrap together (Figure 1) due to high surface energy and surface area and they are used in composite in this bundled form [3]. Compared to the researches done on isolated CNT based composites, there are not much works on CNT bundle based composites. However, there are few studies regarding the orientation of the CNTs in CNT bundle based composites.

From the earlier studies it is seen that there is a wide variation in the reported elastic properties. Reported improvements in the elastic moduli are lower than the expected if the CNTs are assumed to act as reinforcing 


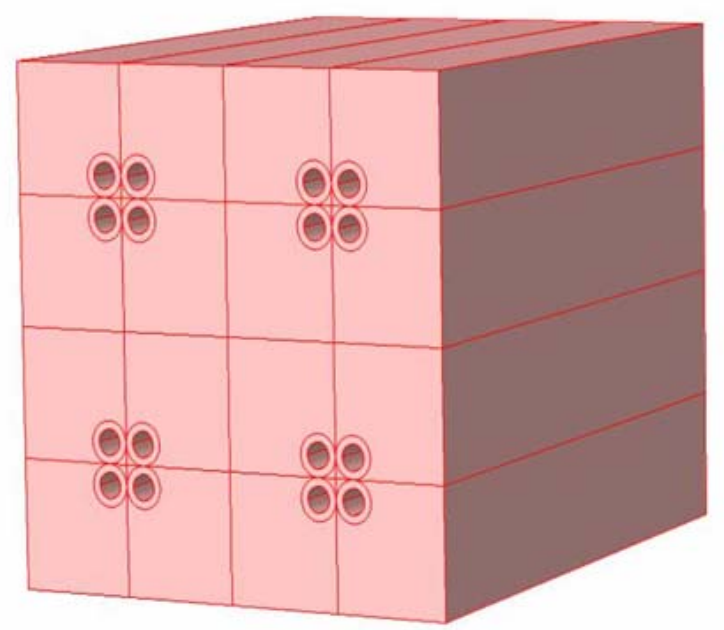

Figure 1. CNT bundle in CNT reinforced CNT-silk compose.

elements with an elastic modulus of $1 \mathrm{TPa}[4,5]$. Discrepancies in the reported elastic moduli as well as in the strength of the CNT based composites may be due to the insufficient load transfer through the interface between the CNT and the polymer matrix of the composites. Load transfer through the interface is affected by several factors. One of the vital factors is the morphology of the CNT bundle (i.e., agglomerated CNTs). Therefore it is necessary to investigate the effect of the morphology of the CNT bundle on the mechanical behavior of the CNT bundle based composites to fully realize the potentials of the CNT-based composites in real engineering applications.

Here a model of four CNTs bundled together is taken into account. Each of the CNT has its own Representative Volume Element (RVE). Then the distance among those four CNTs are varied and the effects of this variation are observed. These observations are mainly observed in two ways: stress concentration in interfaces and change in Young's Modulus of composites. The matrix for all the simulations is Silk. Here all the models are developed and simulations are done using COMSOL Multiphysics $4.2 \mathrm{a}$.

\section{Representative Volume Element (RVE)}

Concept of unit cells or representative volume elements (RVEs) which have been applied successfully in the studies of conventional fiber-reinforced composites at the microscale can be extended to study the CNT-based composites at the nanoscale. In the present study 3D nanoscale square RVEs are employed to investigate the various effects on the elastic properties of nanocomposites.

The RVE used for analyzing long CNT bundle based composite (Figure 2) has a length, $L=10 \mathrm{~nm}$. The chiral index of CNT that is used here is $(10,10)$. The diameter $\left(d_{t}\right)$ of the CNT is obtained from this chiral index [6]. We assume that the volume fraction of our model is $5 \%$. Then using Equation (5) we can get the value of width (a) of the RVE for these diameter and volume fraction.

Four individual RVE models are built and agglomerated together to make the final model (Figure 3). The intersection of the horizontal and vertical line in Figure 3 is taken as the reference point. The distance between this reference point and center of the CNT is varied gradually. Here four cases are discussed where the distances between reference point and center of the CNT are 1.202 nm (Case 1), $2.088 \mathrm{~nm}$ (Case 2), $2.974 \mathrm{~nm}$ (Case 3), 3.86 $\mathrm{nm}$ (Case 4).

\subsection{Calculation of CNT Radius}

The value of the chiral index $(m, n)$ are used in the Equa-

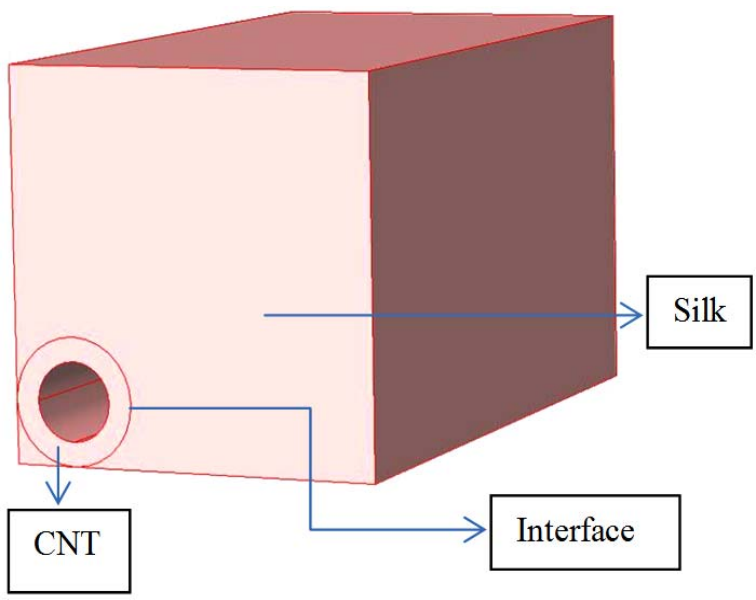

Figure 2. A Representative Volume Element (RVE) with CNT, silk (matrix) and interface.

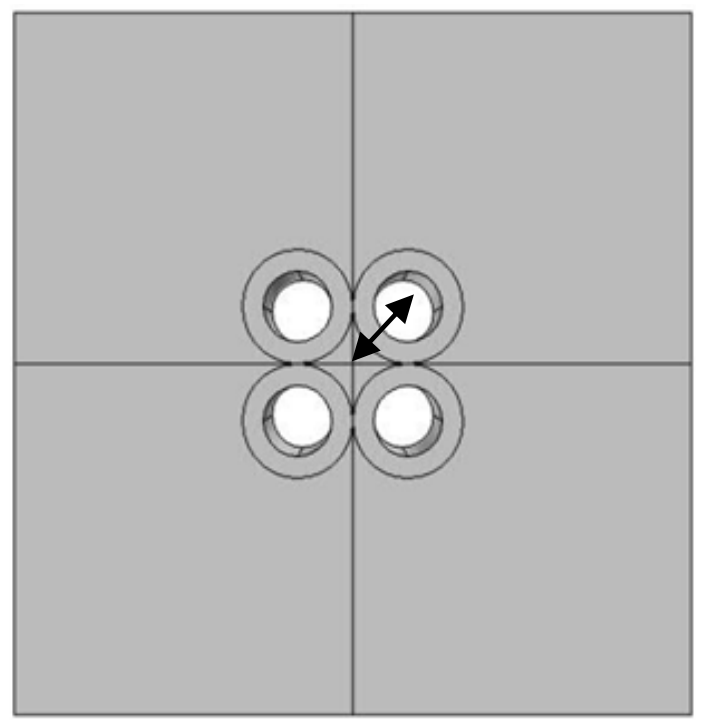

Figure 3. Fraction of the composite to determine its characteristics (the intersection of the horizontal and vertical line being the reference point). 
tion (1) and we can get the value of mean radius of the CNT [6]. Then using Equation (2) we can get the outer radius $\left(r_{0}\right)$ and inner radius $\left(r_{i}\right)$. The chiral index of the CNT taken into account here is $(10,10)$

$$
\begin{gathered}
r_{\text {mean }}=\frac{\sqrt{3} \times 0.144 \times \sqrt{n^{2}+m^{2}+m n}}{2 \pi} \\
r_{0}-r_{i}=0.34 \\
r_{0}=r_{\text {mean }}+0.17 \\
r_{i}=r_{\text {mean }}-0.17
\end{gathered}
$$

\subsection{Volume Fraction of Long CNT in CNT-Matrix Composite}

In case of long CNT based composite, the CNT is relatively long (with large aspect ratio) and it is throughout the RVE. For the square RVE, the volume fraction of the CNT (a tube) is defined by the following equation [7].

$$
V^{t}=\frac{A_{f} L}{A_{c} L}
$$

or

$$
V^{t}=\frac{\pi\left(r_{0}^{2}-r_{i}^{2}\right)}{a^{2}-\pi r_{i}^{2}}
$$

where $A_{f}$ is the cross sectional area of the CNT, $A_{c}$ is the cross sectional area and $\mathrm{L}$ is the length of the composite. In this paper the volume fraction is always kept $5 \%$. So in Equation (5) we put the value of $r_{0}, r_{i}$ (from previous calculation) and volume fraction and thus get the value of the width (a) of the square RVE.

\section{Material Properties}

There are different kinds of silk available in nature. They differ from each other in case of both structural and mechanical properties. Many researches have been done to evaluate these properties. Ko et al. [8] evaluated mechanical properties of different kinds of silk. Some of the silk with their Young's Modulus are Argiope aurentia spider silk (34 GPa), N. clavipe Spider Silk (12.71 GPa), B. Mori Silk (9.90 GPa) [9]. Here for this simulation, the Young's Modulus for silk is taken as $10 \mathrm{GPa}$ which is similar to B. Mori Silk. The Young's Modulus for CNT is taken as $1000 \mathrm{GPa}[4,5]$.

\section{Results and Discussions}

Stress variations on Interfaces, CNTs and Matrix are observed with the variation of the distances between CNTs. Also the variations in Young's Modulus are observed.

A typical figure of COMSOL $4.2 \mathrm{a}$ is represented in Figure 4 where the legends indicating color ranges.

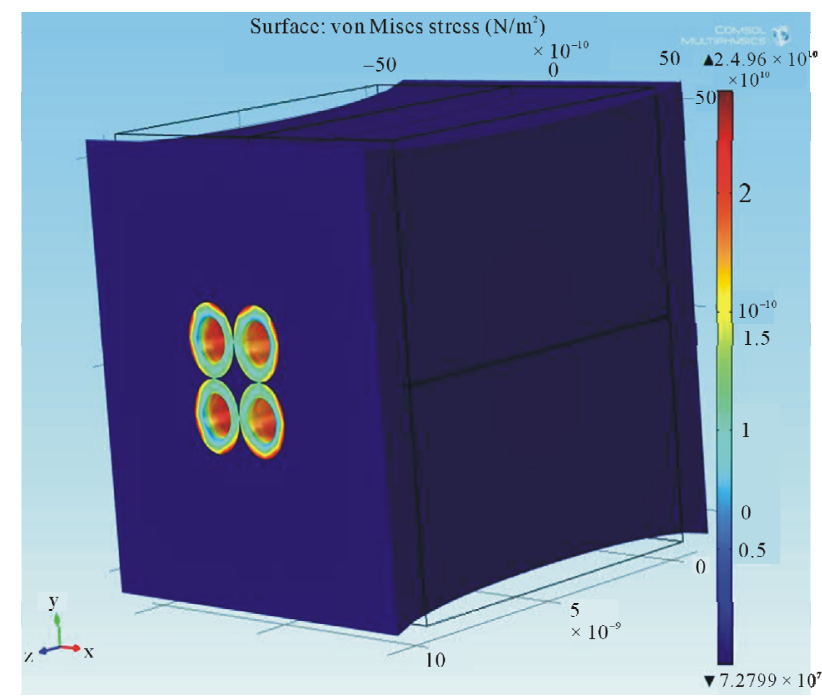

Figure 4. Stress distribution when displacement is along longitudinal direction (z-direction).

\subsection{Effects of Changing the Distances among the CNTs on the Interface, CNT and Matrix}

After making the model, displacement is given on both sides of the structure. This displacement is given along longitudinal direction (z-direction). The stress distribution in the CNTs, Interfaces and Matrix are observed.

In Case 1, the CNTs are actually agglomerated together, so the circumferences of CNTs actually touch each other.

From Figures 5-8, it is evident that highest stress is concentrated at interface between CNT and Matrix (red zone between CNT and Matrix). So this area has the highest possibility of failure. These are indicated with red circumferential areas. Also as the distance between CNTs increases, these areas get reduced. That means, as the distance between CNTs are increasing, the possibility of interfacial failure will reduce. This is evident in Figures 5 and 6. It can be seen that the red circumferential area around the CNT reduces as much as half in Figure 6 than Figure 5.

Stress is also concentrated in CNT but the matrix shows less sign of stress. Because the Young's Modulus of the CNT (1000 GPa) is much higher than that of Silk (Matrix). So CNTs carry most of the load.

The variation of stress along the length $(10 \mathrm{~nm})$ of interface, CNT and Matrix is showed for all the four cases in Figures 9-12.

It can be seen that the stress variation in matrix is very low.

The stress of the matrix is lowest among Interface, CNT and Matrix. It has an average value of $200 \mathrm{MPa}$. The variation of stress along the length of CNT is almost same for all three cases. The most notable thing that is observed in these graphs is that the highest stress (24.6 $\mathrm{GPa}$ ) in interface can be observed for Case 1. As the dis- 


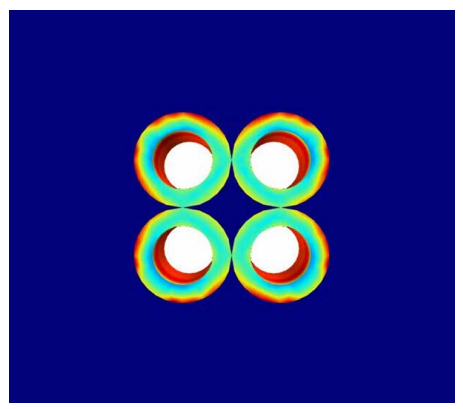

Figure 5. Stress distribution in CNT-silk composite (when the distance between center of the CNT and reference point being $1.202 \mathrm{~nm}$ ) for Case 1.

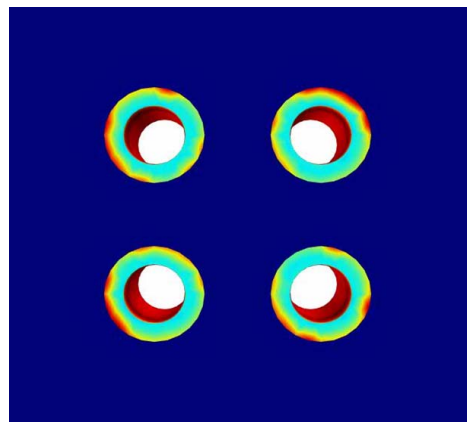

Figure 6. Stress distribution in CNT-silk composite (when the distance between center of the CNT and reference point being $2.088 \mathrm{~nm}$ ) for Case 2 .

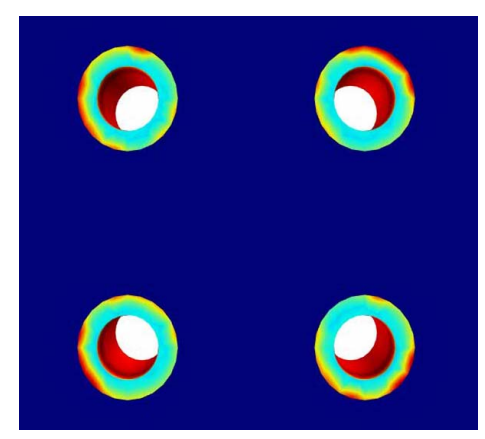

Figure 7. Stress distribution in CNT-silk composite (when the distance between center of the CNT and reference point being $2.974 \mathrm{~nm}$ ) for Case 3 .

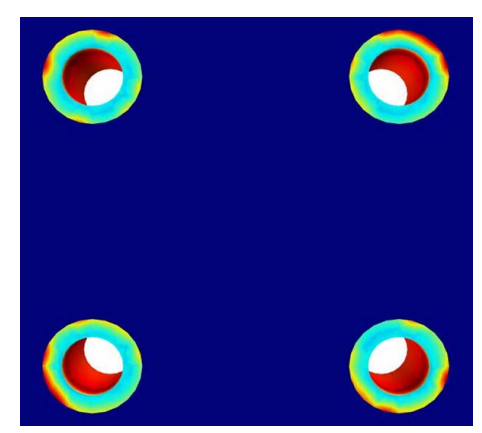

Figure 8. Stress distribution in CNT-silk composite (when the distance between center of the CNT and reference point being $3.86 \mathrm{~nm}$ ) for Case 4 .

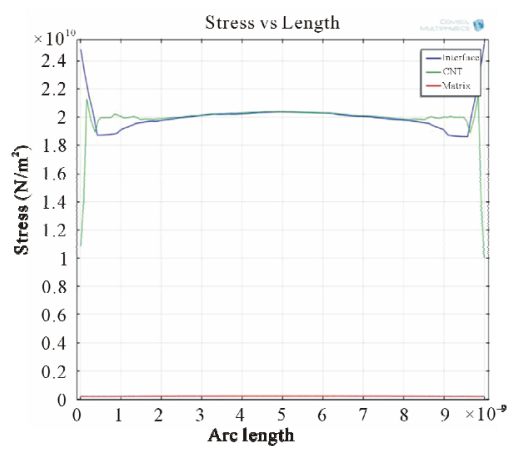

Figure 9. Variation of stress along the length of Interface, CNT and Matrix for Case 1.

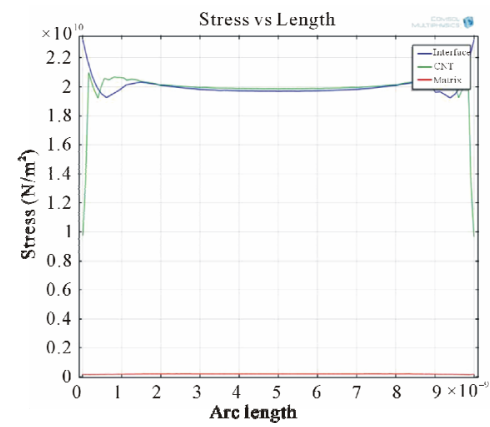

Figure 10. Variation of stress along the length of Interface, CNT and Matrix for Case 2.

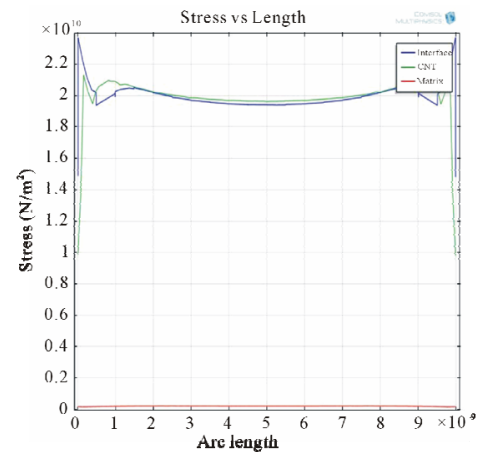

Figure 11. Variation of stress along the length of Interface, CNT and Matrix for Case 3.

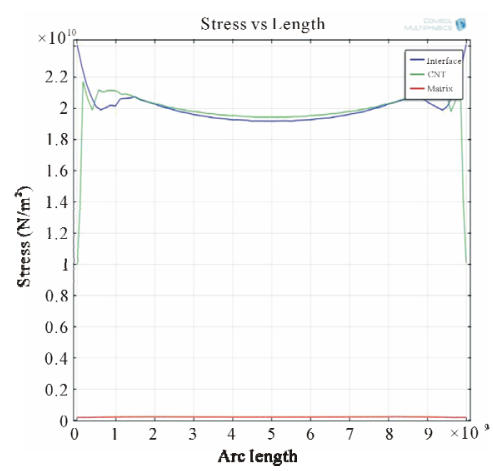

Figure 12. Variation of stress along the length of Interface, CNT and Matrix for Case 4. 
tances between the CNTs are increased, the stress at Interfaces decreases.

The variation in stress is also observed when the displacement was given in transverse direction ( $x$-direction).

Here it is seen that the stress is concentrated in the inner radius of the CNT and it is along the $x$-direction. In Figures 13 and 14 it is showed that much variation in stress concentration is not observed when the distances between CNTs are changed.

The approach here is to give displacement and observe the variation of stress concentration at Interface, CNT and matrix. There is another approach where load will be applied on both ends and then the variation of the displacement can be observed. A typical example of that approach is showed in Figure 15.

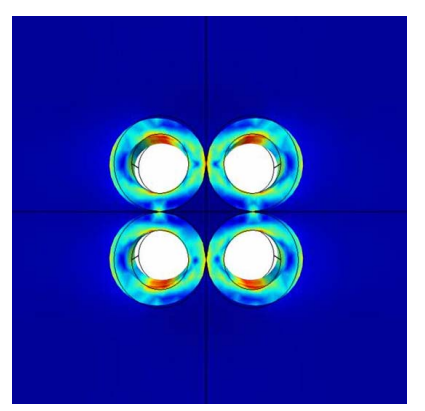

Figure 13. Stress distribution in CNT-silk composite (when the distance between center of the CNT and reference point being $1.202 \mathrm{~nm}$ ) for Case 1.

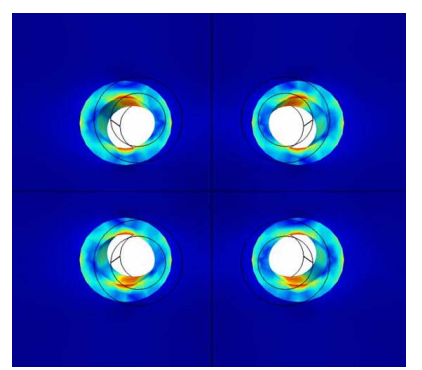

Figure 14. Stress distribution in CNT-silk composite (when the distance between center of the CNT and reference point being $2.088 \mathrm{~nm}$ ) for Case 2 .

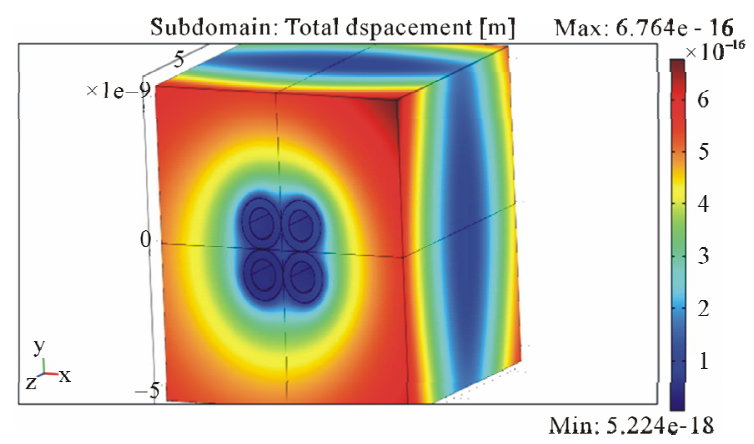

Figure 15. Total displacement in matrix when load is along longitudinal direction (z-direction).

\subsection{Variation in Young's Modulus for Different Orientations of the CNT}

As described earlier, there is change in stress distribution when the distances between the CNTs are varied. There is also variation in Young's Modulus as the distance between the reference point and center of the CNT is varied. Here also to describe this, four cases are taken into account where the distances are: $1.202 \mathrm{~nm}$ (Case 1), 2.088 nm (Case 2), $2.974 \mathrm{~nm}$ (Case 3), $3.86 \mathrm{~nm}$ (Case 4).

The Longitudinal Young's Modulus decreases from 84.9 $\mathrm{GPa}$ to $74.9 \mathrm{GPa}$ when the distance increases from $1.202 \mathrm{~nm}$ to $3.86 \mathrm{~nm}$ (Figure 16).

The Transverse Young's Modulus increases from 14.05 $\mathrm{GPa}$ to $14.5 \mathrm{GPa}$ when the distance increases from $1.202 \mathrm{~nm}$ to $3.86 \mathrm{~nm}$ (Figure 17).

\section{Conclusions}

The conclusion can be summarized as:

1) If the distances between CNTs are increased, stress at Interfaces reduces when the displacement is along longitudinal direction. So with the increment of distances

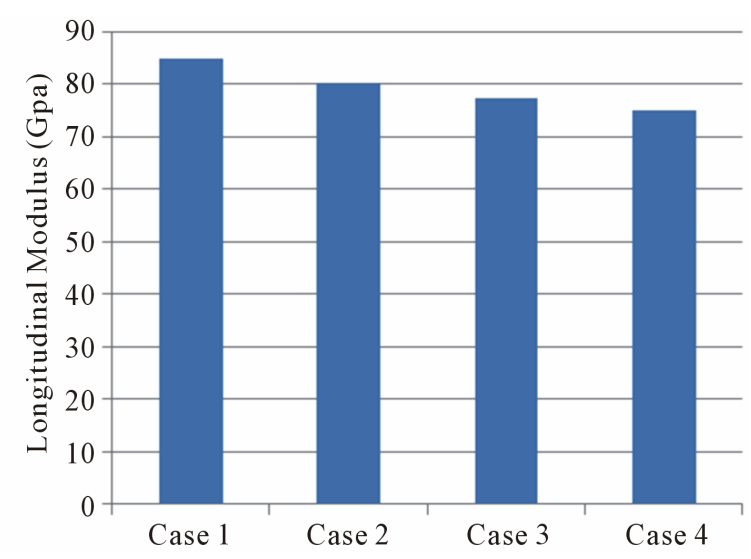

Figure 16. Changes in Longitudinal Young's Modulus for different cases.

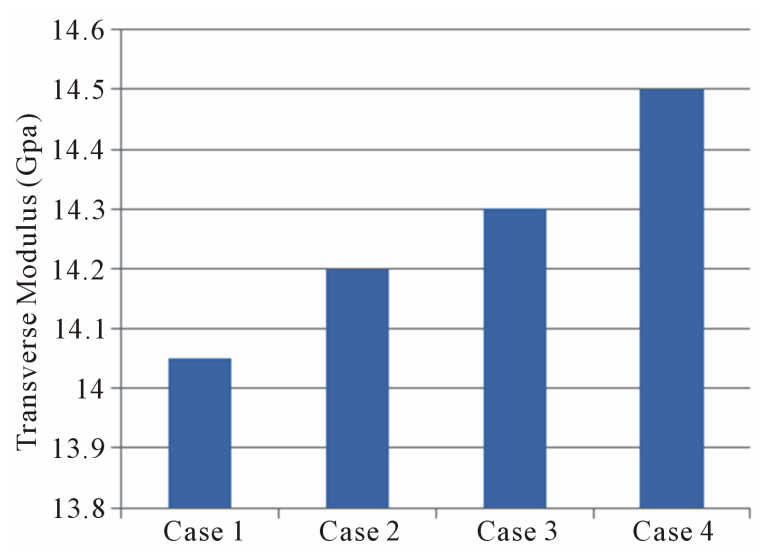

Figure 17. Changes in Transverse Young's Modulus for different cases. 
between CNTs, there is less possibility of failure.

2) There is not much difference in stress distribution at Interface, CNT and matrix when the displacement is along transverse direction.

3) Longitudinal Young's Modulus decreases with the increment of the distance between CNTs.

4) Transverse Young's Modulus increases with the increment of the distance between CNTs.

\section{REFERENCES}

[1] L. H. Peebles, "Carbon Fibers: Formation, Structure and Properties," CRC Press, Boca Raton, 1995.

[2] C. Le, S. Soni, E. Y.-T. Chen and W. C. Chin, "SilkCarbon Nanotube Composite for Stem Cell Neuronal Differentiation," Nanoelectronics Conference (INEC), TaoYuan, 21-24 June 2011, pp. 1-2.

[3] E. W. Wong, P. E. Sheehan and C. M. Liever, "Nanobeam Mechanics: Elasticity, Strength and Toughness of Nanorods and Nano Tubes," Science, Vol. 227, No. 5334, 1997, pp. 1971-1975. doi:10.1126/science.277.5334.1971

[4] P. Zhang, Y. Huang, P. H. Geubelle, P. A. Klei and K. C. Hwang, "The Elastic Modulus of Single-Wall Carbon
Nanotubes: A Continuum Analysis Incorporating Interatomic Potentials," International Journal of Solids and Structures, Vol. 39, No. 13-14, 2002, pp. 3893-3906.

[5] A. Krishnan, E. Dujardin, T. W. Ebbesen, P. N. Yianilos and M. M. J. Treacy, "Young's Modulus of Single Walled Nanotubes," Physical Review B, Vol. 58, No. 20, 1998, pp. 14013-14019. doi:10.1103/PhysRevB.58.14013

[6] B. R. Pipes, S. J. V. Frankland, P. Hubertc and E. Saether, "Self-Consistent Properties of Carbon Nanotubes and Hexagonal Arrays as Composite Reinforcements," Composites Science and Technology, Vol. 63, No. 10, 2003, pp. 1349-1358. doi:10.1016/S0266-3538(03)00020-4

[7] X. L. Chen and Y. J. Liu, "Square Representative Volume Elements for Evaluating the Effective Material Properties of Carbon Nanotube-Based Composite," Computational Materials Science, Vol. 29, No. 1, 2004, pp. 1-11. doi:10.1016/S0927-0256(03)00090-9

[8] F. K. Ko, S. Kawabata, M. Inoue, M. Niwa, S. Fossey and J. W. Song, "Engineering Properties of Spider Silk," 2004. http://web.mit.edu/course/3/3.064/www/slides/Ko_spider silk.pdf

[9] M. J. Bonino, "Material Properies of Spider Silk," University of Rochester, Rochester, 2003. 